\title{
TWO PROBLEMS IN QUANTIZED ALGEBRAS OF FUNCTIONS
}

\author{
YA.S.SOIBELMAN
}

1. Let $C(K)_{q}$ be the algebra of functions which are continuous on compact quantum group $K$ (see [1] for definition). It is well known from [1] that there is an imbedding of $C(K)_{q}$ into the algebra of continuous operator-functions on the maximal torus $T \subset K$. Describe the image. The answer is known for $K=S U(2)$ (see [2]). There is such a description for odd-dimensional quantum spheres (see [3]).

2. Let $g$ be a finite dimensional complex Lie algebra, $C\left[g^{*}\right]$ be an algebra of polynomial functions on dual space $g^{*}$. Let us equip $C\left[g^{*}\right]$ with Poisson brackets by using the Lie-Kirillov formulas. Quantization of this Hopf-Poisson algebra is known: it is the universal enveloping algebra $U(g)$. Therefore the usual method of orbit (KonstantKirillov) gives rise to the relation between the representation theory of the algebra of functions on the quantum group $g^{*}$ and symplectic leaves in the Poisson-Lie group $g^{*}$ (these are coadjoint orbits in our case).

Problem. How to generalize this to the case of more general quantum groups?

I mean the generalization of the method of orbits. The correspondence between representations and leaves was investigated in $[1,2]$ for compact quantum groups.

\section{References}

[1] Ya.Soibelman, Algebra of functions on compact quantum group and its representations., Algebra Anal 2 no. 1 (1990), 190-212. (in Russian)

[2] L.Vaksman, Ya.Soibelman, Algebra of functions on quantutm group SU(2), Funkz. Anal. Pril. 22 no, 3 (1988), 1-14. (in Russian)

[3] L.Vaksman, Ya.Soibelman, Algebra of functions on quantum group $S U(n+1)$ and odd-dimensional quantum spheres, Algebra Anal. 2 no. 5 (1990), 101-120. (in Russian) 